\title{
Influence of space charges on the current-voltage characteristic of organic light-emitting devices
}

\author{
H. Riel a, *, W. Brütting ${ }^{\text {b }}$, T. Beierlein ${ }^{\text {a }}$, E. Haskal ${ }^{\text {a }}$, P. Müller ${ }^{\text {a }}$ W. Rieß ${ }^{\text {a }}$ \\ ${ }^{\text {a } I B M}$ Research, Zurich Research Laboratory, 8803 Rüschlikon, Switzerland \\ ${ }^{\mathrm{b}}$ Experimental Physics II, University of Bayreuth, 95440 Bayreuth, Germany
}

\begin{abstract}
The role of space charges on the device characteristics of multilayer organic light-emitting devices (OLEDs) is investigated. We studied OLEDs consisting of copper phthalocyanine $(\mathrm{CuPc})$ as buffer and hole injection layer, $N, N^{\prime}$-di(naphthalene-1-yl)- $N, N^{\prime}$-diphenylbenzidine (NPB) as hole transport layer, and tris(8-hydroxyquinolinato)aluminum $\left(\mathrm{Alq}_{3}\right)$ as electron transport and emitting layer sandwiched between high and low work function metal electrodes.

Detailed current-voltage measurements show that the device characteristics at low bias depend strongly on sweep direction as well as on sweep speed, indicating that space charges accumulate within the organic layers. On the one hand these space charges increase the electric field for electron injection at the cathode, on the other hand they screen the applied electrical field and thus determine the steepness of the current-voltage characteristics. Reducing these space charges by fabricating optimized structures where the limiting interfaces between the different organic layers are graded results in a significantly enhanced current flow and higher brightness at a given voltage.
\end{abstract}

Keywords: Organic light-emitting diodes; Space charges; Graded interfaces; Internal barriers

\section{Introduction}

Organic light emitting diodes (OLEDs) have been the subject of intense investigation in the past few years because of their potential applications as emissive elements for flat panel color displays [1-8]. Long life, low power consumption and low operating voltage are essential in such applications. Therefore huge efforts have gone into fabricating more efficient and stable devices. Meanwhile, luminous efficiencies of green OLEDs exceed $10 \mathrm{~lm} / \mathrm{W}$ [9], and lifetimes of more than $80,000 \mathrm{~h}$ at a starting brightness of $100 \mathrm{~cd} / \mathrm{m}^{2}$ seem to be attainable [10,11]. For display applications, especially for active matrix addressing, the operating voltage should be as low as possible. In recent years tremendous progress has been achieved in reducing the voltage mainly by optimizing and modifying the anode and cathode electrodes [12-14] and by using new concepts for charge carrier injection, like inorganic

\footnotetext{
${ }^{*}$ Corresponding author. Tel.: +41-1-724-8334; fax: +41-1-724-8956. E-mail address: hei@zurich.ibm.com (H. Riel).
}

injection layers [15-18] as well as inorganic/organic multilayer device structures [19].

However, not only the energy barriers at the charge carrier injecting electrodes are important, the barriers between the different organic layers of a multilayer OLED could also affect the device characteristics. These internal barriers lead together with the different charge carrier mobilities in adjacent organic layers to the accumulation of space charges at those interfaces. Consequently, the applied electrical field is screened, resulting in a flatter device characteristics and a reduced current flow at a given voltage. Besides that, these space charges may also lead to an enhanced degradation of the device. For optimized performance and improved device characteristics, it is essential to understand the critical interfaces and to avoid/reduce these space charges by thorough device design. In this paper, we describe the influence of space charges on the device characteristics of a multilayer OLED, and introduce the simple concept of graded interfaces between the different organic layers in order to prevent these space charges and thus being able to achieve significant enhanced current flow and higher brightness compared to the conventional OLED at the same voltage. 


\section{Experimental}

The OLEDs were built on glass substrates (Schott AF45) precoated with an opaque high workfunction metal anode. The organic multilayer structure consists of copper phthalocyanine (CuPc), $N, N^{\prime}$-di(naphthalene-1-yl)- $N, N^{\prime}$ diphenyl-benzidine (NPB) and tris(8-hydroxyquinolinato)aluminum $\left(\mathrm{Alq}_{3}\right)$ with typical thicknesses of $20 \mathrm{~nm}$, $45 \mathrm{~nm}$ and $60 \mathrm{~nm}$, respectively, and a thin Ca cathode (20 $\mathrm{nm}$ ) on top. Electroluminescence (EL) in this configuration is observed through the semitransparent cathode. The active area of our devices is $2 \times 3 \mathrm{~mm}^{2}$.

Prior to use, all organic materials were purified by vacuum sublimation. Depositions were carried out in a high vacuum system (Leybold) by thermal evaporation from resistively heated tantalum and tungsten boats. The base pressure in the chamber ranged between $4 \times 10^{-7}$ and $1 \times 10^{-6}$ mbar. The typical deposition rate for the organic compounds and the metal was $\approx 1 \AA / \mathrm{s}$. Graded interfaces of the different organic layers were obtained by coevaporation. Deposition rates were individually controlled by calibrated quartz-crystal monitors. Since the evaporation system is equipped with a rotating sample holder and a specially designed shutter mechanism, three devices can simultaneously be fabricated and independently controlled. This enables side-by-side comparison of OLEDs differing in only one parameter and thus uncertainties are avoided, which would result from different evaporation processes. The growth chamber is attached directly to a glove box system filled with argon, which allows to fabricate, characterize, and encapsulate devices completely under inert conditions.

Current-voltage $(I-V)$ and electroluminescence-voltage $(\mathrm{EL}-V)$ characteristics were measured with a Hewlett Packard parameter analyzer (HP 4145B) and a sensitive Si photodiode (Hamamatsu S2281). Calibrated brightness measurements were obtained with a Photo Research PR 704 spectroradiometer.

\section{Results and discussion}

Fig. 1 shows the dependence of the $I-V$ and EL- $V$ characteristics on the sweep direction of an anode $/ \mathrm{CuPc} / \mathrm{NPB} / \mathrm{Alq}_{3} / \mathrm{Ca}$ device. In order to reveal the influence of different sweep directions on the device characteristics the data are presented in a semi-logarithmic plot. The " - to +" voltage sweep started at -5 to $10 \mathrm{~V}$ in $50 \mathrm{mV}$ steps and the " + to - " sweep was measured in reverse direction. In order to guarantee similar sweep speed in both cases, the integration time of the parameter analyzer and the delay time between individual data points was set identical (medium, $0 \mathrm{~s}$ ). In this mode the acquisition time for a single data point depends on the magnitude of the detected current and is around $100 \mathrm{~ms}$ for values below $10 \mathrm{nA}$. In Fig. 1, the current increases sharply in

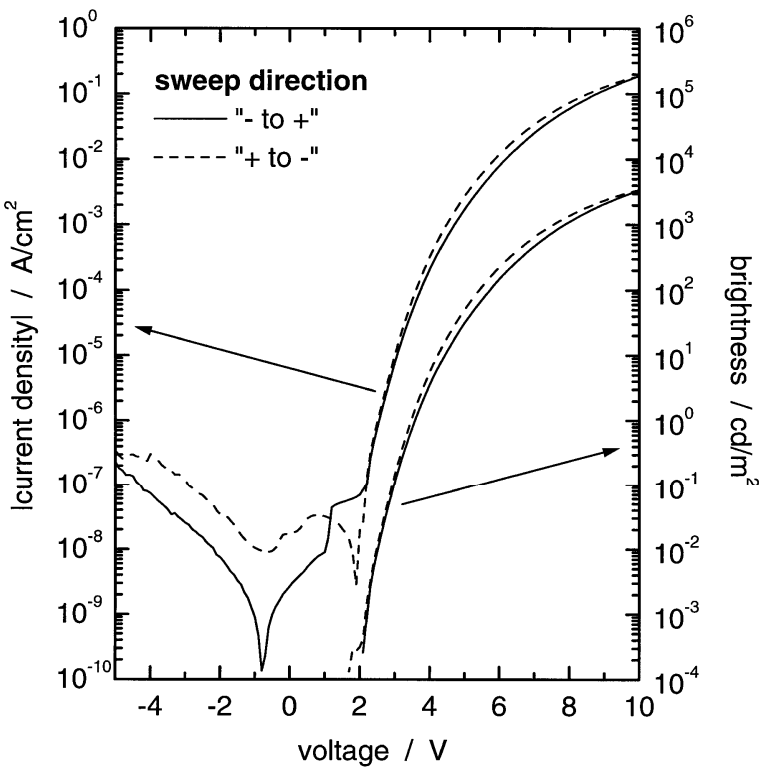

Fig. 1. Influence of the sweep direction on the $I-V$ and EL- $V$ characteristics of the same anode $/ \mathrm{CuPc} / \mathrm{NPB} / \mathrm{Alq}_{3} / \mathrm{Ca}$ OLED measured with identical acquisition time.

forward bias above a threshold voltage of $2 \mathrm{~V}$ for both sweep directions and shows only slight differences in the medium voltage range. However, a pronounced hysteresis occurs in the range from -5 to $2 \mathrm{~V}$. The onset voltage of electroluminescence defined by $10^{-3} \mathrm{~cd} / \mathrm{m}^{2}$ is $2.1 \mathrm{~V}$ and is not influenced by the measurement parameters. Below 2 $\mathrm{V}$, this is the range where only one type of charge carrier (holes) is injected, a strong dependence on the measurement direction is observed. In particular, the voltage where the zero crossing of current occurs is not at zero bias. Starting from negative voltages it appears at around -0.8 $\mathrm{V}$, and at about $+1.9 \mathrm{~V}$ when sweeping vice versa. Additionally, the current in the " - to +" voltage sweep shows a pronounced structure at voltages between 1 and 2 $\mathrm{V}$, which cannot be attributed to leakage current. The hole current increases steeply at around $1 \mathrm{~V}$ and flattens abruptly. The slight hysteresis in the medium voltage range occurs in the current as well as in the EL signal and we find that the hysteresis is more pronounced if higher forward voltages are applied.

Not only the sweep direction has significant influence on the device characteristics below $2 \mathrm{~V}$ but also the measurement speed. Fig. 2 shows the $I-V$ and EL- $V$ characteristics of the same device shown in Fig. 1, however, measured with different acquisition times. The solid and dashed lines were obtained with integration time short and long, which corresponds to an acquisition time in the range of $50 \mathrm{~ms}$ and $1 \mathrm{~s}$, respectively. The dotted line was measured with integration time long and an additional delay time of $3 \mathrm{~s}$ between individual data points was set. All three measurements started at $-5 \mathrm{~V}$.

We observe an almost identical device characteristics independent of the sweep speed for voltages above the 


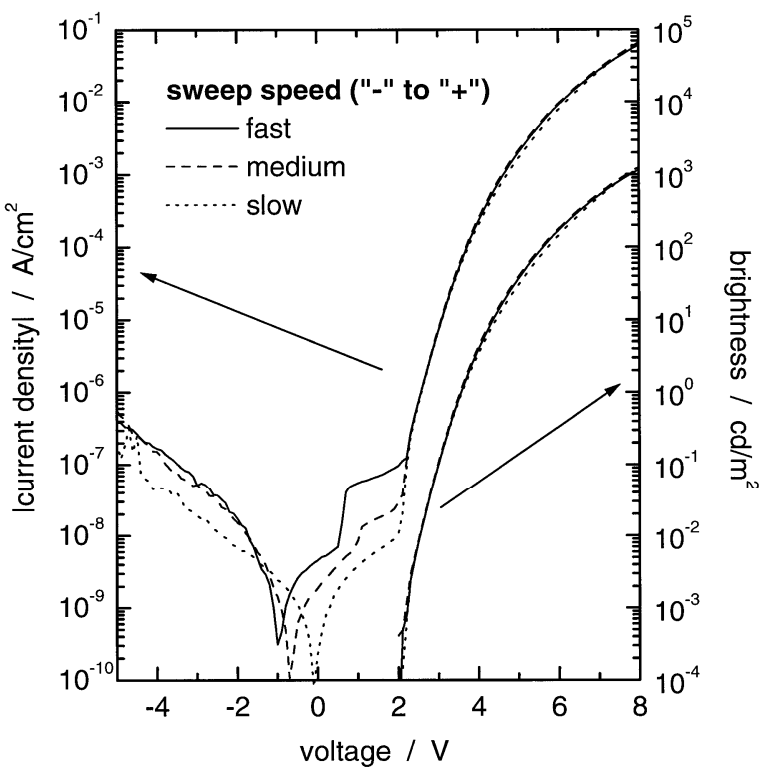

Fig. 2. $I-V$ and EL- $V$ characteristics of the device shown in Fig.1 measured with different sweep speeds (sweep direction: " - to + "').

onset of EL. However, below $2 \mathrm{~V}$, major differences in the $I-V$ characteristics appear. In particular, the zero crossing of the current depends strongly on the measurement speed. With decreasing sweep velocity the zero crossing voltage shifts from -1.0 to -0.7 to $-0.05 \mathrm{~V}$. Using even longer delay times the zero crossing of the current takes place at exactly $0 \mathrm{~V}$. In addition, the pronounced structure in the current at voltages between 0 and $2 \mathrm{~V}$ is also significantly affected by the measurement time. With longer acquisition times the sharp increase in current just above $0 \mathrm{~V}$ disappears.

These observations clearly indicate the presence of space charges in the multilayer OLED. In fact detailed investigations of the $C-V$ characteristics and careful static charge measurements have revealed their existence [20]. As mentioned before, the internal energy barriers together with the different charge carrier mobilities in the various organic materials can lead to accumulation of space charges at the organic-organic interfaces. Since the observed phenomena occur mainly in the voltage range where only holes are injected into the multilayer structure the energy barriers between $\mathrm{CuPc}$ and NPB as well as between NPB and $\mathrm{Alq}_{3}$ can be responsible for these effects.

In order to find the limiting barrier we fabricated OLEDs with graded interfaces and compared their device characteristics with the conventional multilayer structure (see Fig. 3). The $I-V$ and EL- $V$ curves were measured with identical parameters starting at $-5 \mathrm{~V}$. Apart from slight variations in the zero crossing of the current all three devices show an almost identical behavior in reverse direction. Moreover their onset voltage of EL $(2.1 \mathrm{~V})$ is nearly identical. However, above this threshold voltage the OLEDs with graded interfaces show a much steeper characteristics and therefore a higher current flow accompanied by an enhanced brightness at a given voltage. For example, whereas the conventional structure requires $6.0 \mathrm{~V}$ to obtain $100 \mathrm{~cd} / \mathrm{m}^{2}$, the CuPc-NPB graded OLED needs only 5.0 $\mathrm{V}$. The steeper characteristics of the devices with the graded interfaces can be based on several effects. First of all, the regions where charge carriers can accumulate are smeared out, and thus the internal space charge is redistributed. This, in turn, leads to a modified internal electrical field distribution and therefore to an enhanced probability for holes to enter the adjacent layer. In addition, due to the disorder the density of states of both materials in the graded region is broadened which facilitates the injection process. As a consequence the influence of the internal barriers is reduced, explaining the significantly steeper $I-V$ and $\mathrm{EL}-V$ characteristics.

Comparing the two devices with the graded interface it is conspicuous that the CuPc-NPB graded OLED shows the steepest $I-V$ characteristics and the highest brightness. Furthermore, while the pronounced structure between 0 and $2 \mathrm{~V}$ is observed in the normal and the NPB-Alq graded OLED, it is significantly reduced in the $\mathrm{CuPc}-\mathrm{NPB}$ graded device. This indicates that the abrupt $\mathrm{CuPc}-\mathrm{NPB}$ interface is the dominating barrier in the conventional structure where most of the holes accumulate.

To summarize, our studies have clearly shown that positive space charges which accumulate at the $\mathrm{CuPc}-\mathrm{NPB}$ and $\mathrm{NPB}-\mathrm{Alq}_{3}$ interface limit the injection into the adjacent organic layer. Therefore internal barriers rather than charge carrier transport restrict the current flow and determine the device characteristics of multilayer OLEDs.

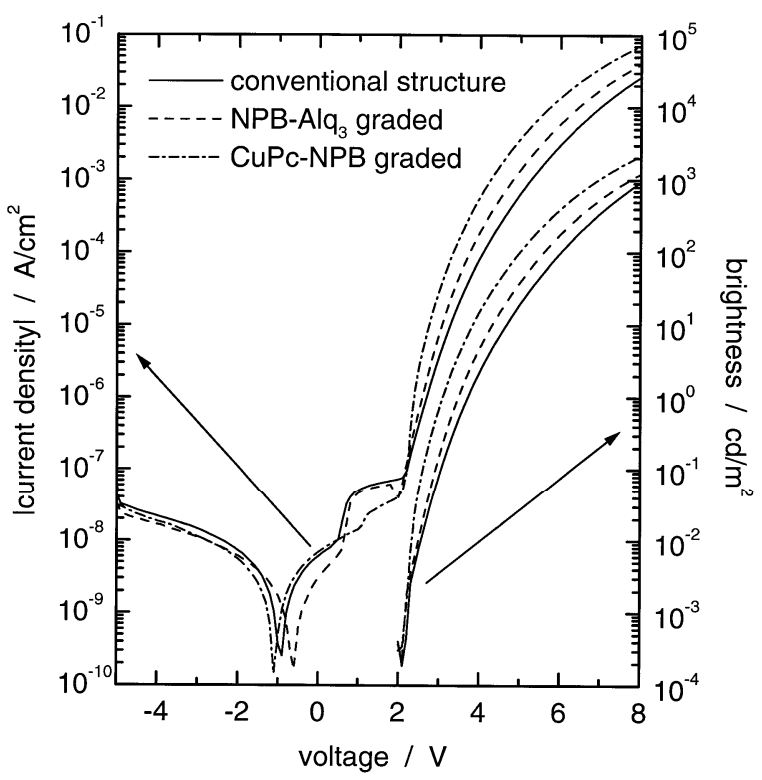

Fig. 3. Comparison of the $I-V$ and EL $-V$ characteristics of three OLEDs differing in only one parameter. Their structures are anode $/ \mathrm{CuPc} /$ $\mathrm{NPB} / \mathrm{Alq}_{3} / \mathrm{Ca}$, anode/CuPc/NPB /NPB: $\mathrm{Alq}_{3} / \mathrm{Alq}_{3} / \mathrm{Ca}$, and anode $/ \mathrm{CuPc} / \mathrm{CuPc}: \mathrm{NPB} / \mathrm{NPB} / \mathrm{Alq}_{3} / \mathrm{Ca}$, respectively. 


\section{Acknowledgements}

We thank Meinrad Tschudy for preparing the substrates.

\section{References}

[1] C.W. Tang, S.A. VanSlyke, Appl. Phys. Lett. 51 (1987) 913.

[2] C. Adachi, T. Tsutsui, S. Saito, Appl. Phys. Lett. 56 (1990) 799.

[3] J. Kido, M. Kimura, K. Nagai, Science 267 (1995) 1332.

[4] T. Wakimoto, R. Murayama, K. Nagayama, Y. Okuda, H. Nakada, T. Tohma, in: J. Morreale (Ed.), SID Int'l Symp. Digest of Technical Papers Vol. 27 Society for Information Display, Santa Ana, CA, 1996, p. 849

[5] H. Nakada, T. Tohma, in: R.H. Mauch, H.-E. Gumlich (Eds.), Proc. Int'l Symp. on Inorganic and Organic Electroluminescence, Wissenschaft und Technik, Berlin, 1996, p. 385.

[6] H. Nakamura, C. Hosokawa, T. Kusumoto, in: R.H. Mauch, H.-E. Gumlich (Eds.), Proc. Int'l Symp. on Inorganic and Organic Electroluminescence, Wissenschaft und Technik, Berlin, 1996, p. 95.

[7] Y. Hamada, T. Sano, H. Fujii, Y. Nishio, H. Takahashi, K. Shibata, Jpn. J. Appl. Phys. 35 (1996) 1339.

[8] S. Miyata, H.S. Nalwa (Eds.), Organic Electroluminescent Materials and Devices, Gordon and Breach, Amsterdam, 1997.

[9] T. Sano, Y. Hamada, K. Shibata, in: R.H. Mauch, H.-E. Gumlich
(Eds.), Proc. Int'l Symp. on Inorganic and Organic Electroluminescence, Wissenschaft und Technik, Berlin, 1996, p. 249.

[10] C.W. Tang, Information Display 12 (1996) 16.

[11] C.W. Tang, in: J. Morreale (Ed.), SID Int'l Symp. Digest of Technical Papers Vol. 27 Society for Information Display, Santa Ana, CA, 1996, p. 181.

[12] T. Wakimoto, S. Kawani, K. Nagayama, Y. Yonemoto, R. Murayama, J. Funaki, H. Sato, H. Nakada, K. Imai, in: Technical Digest of the Int'l Symp. on Inorganic and Organic Electroluminescence, Hamamatsu, 1994, p. 77.

[13] S. Fujita, T. Sakamoto, K. Ueda, K. Ohta, S. Fujita, Jpn. J. Appl. Phys. 36 (1997) 350, Part 1

[14] A. Berntsen, P. van de Weijer, Y. Croonen, C. Liedenbaum, J. Vleggaar, in: J. Morreale (Ed.), Conference Record of the 17th Int'l Display Research Conference, Society for Information Display, Santa Ana, CA, 1997, p. F 28.

[15] M. Meier, M. Cölle, S. Karg, E. Buchwald, J. Gmeiner, W. Rieß, M. Schwoerer, Mol. Cryst. Liq. Cryst. 283 (1996) 197.

[16] L.S. Hung, C.W. Tang, M.G. Mason, Appl. Phys. Lett. 70 (1997) 152.

[17] F.G. Celii, S.J. Jacobs, in: J. Morreale (Ed.), Conference Record of the 17th Int'l Display Research Conference, Society for Information Display, Santa Ana, CA, 1997, p. 314.

[18] G.E. Jabbour, Y. Kawabe, S.E. Shaheen, J.F. Wang, M.M. Morrel, B. Kippelen, N. Peyghambarian, Appl. Phys. Lett. 71 (1997) 1762.

[19] W. Rieß, H. Riel, P.F. Seidler, H. Vestweber, Synth. Met. 99 (1999) 213.

[20] W. Brütting et al., in preparation. 